\title{
Non-invasive measurement of pressure gradients using ultrasound
}

\author{
Olesen, Jacob Bjerring; Traberg, Marie Sand; Pihl, Michael Johannes; Jensen, Jørgen Arendt
}

\section{Published in:}

Proceedings of SPIE

Link to article, DOI:

$10.1117 / 12.2006732$

Publication date:

2013

Link back to DTU Orbit

Citation (APA):

Olesen, J. B., Traberg, M. S., Pihl, M. J., \& Jensen, J. A. (2013). Non-invasive measurement of pressure gradients using ultrasound. In Proceedings of SPIE: Ultrasonic Imaging, Tomography, and Therapy (Vol. 8675). SPIE - International Society for Optical Engineering. https://doi.org/10.1117/12.2006732

\section{General rights}

Copyright and moral rights for the publications made accessible in the public portal are retained by the authors and/or other copyright owners and it is a condition of accessing publications that users recognise and abide by the legal requirements associated with these rights.

- Users may download and print one copy of any publication from the public portal for the purpose of private study or research.

- You may not further distribute the material or use it for any profit-making activity or commercial gain

- You may freely distribute the URL identifying the publication in the public portal

If you believe that this document breaches copyright please contact us providing details, and we will remove access to the work immediately and investigate your claim 


\title{
Non-invasive measurement of pressure gradients using ultrasound
}

\author{
Jacob Bjerring Olesen, Marie Sand Traberg, Michael Johannes Pihl and Jørgen Arendt Jensen \\ Center for Fast Ultrasound Imaging, Dept. of Elec. Eng., Bldg. 349, \\ Technical University of Denmark, DK-2800 Kgs. Lyngby, Denmark.
}

\begin{abstract}
A non-invasive method for estimating 2-D pressure gradients from ultrasound vector velocity data is presented. The method relies on in-plane vector velocity fields acquired using the Transverse Oscillation method. The pressure gradients are estimated by applying the Navier-Stokes equations for isotropic fluids to the estimated velocity fields. The velocity fields were measured for a steady flow on a carotid bifurcation phantom (Shelley Medical, Canada) with a 70\% constriction on the internal branch. Scanning was performed with a BK8670 linear transducer (BK Medical, Denmark) connected to a BK Medical 2202 UltraView Pro Focus scanner. The results are validated through finite element simulations of the carotid flow model where the geometry is determined from MR images. This proof of concept study was conducted at nine ultrasound frames per second. Estimated pressure gradients along the longitudinal direction of the constriction varied from $0 \mathrm{kPa} / \mathrm{m}$ to $10 \mathrm{kPa} / \mathrm{m}$ with a normalized bias of $-9.1 \%$ for the axial component and $-7.9 \%$ for the lateral component. The relative standard deviation of the estimator, given in reference to the peak gradient, was $28.4 \%$ in the axial direction and $64.5 \%$ in the lateral direction. A study made across the constriction was also conducted. This yielded magnitudes from $0 \mathrm{kPa} / \mathrm{m}$ to 7 $\mathrm{kPa} / \mathrm{m}$ with a normalized bias of $-5.7 \%$ and $13.9 \%$ for the axial and lateral component, respectively. The relative standard deviations of this study were $45.2 \%$ and $83.2 \%$ in the axial and lateral direction, respectively.
\end{abstract}

Keywords: Medical ultrasound, Pressure gradients, Flow, Stenosis, Navier-Stokes equation

\section{INTRODUCTION}

Local pressure gradients in hemodynamics provide important information for diagnosing various cardiovascular diseases such as atherosclerosis. ${ }^{1}$ The gradients are used as an indication on how changes in the flow caused by plaque formation affects the risk of embolism. Today, pressure gradients are measured by means of catheters inserted into the femoral artery and threaded to the region of interest. Although this procedure is reported reliable and of low risk, ${ }^{2}$ it remains an invasive procedure that exposes the patients to ionizing radiation during angiography guidance. Furthermore, the presence of the catheter causes inconvenient disturbances to the surrounding fluid flow, thereby affecting the pressure field it is measuring. A less invasive method for measuring the local pressure gradients was proposed by Fairbank and Scully. ${ }^{3}$ The method relies on injecting contrast agent microbubbles into the circulatory system and measure the frequency shift in the scattered spectrum as ultrasonic waves are applied. A range of other methods for estimating pressure changes has also been devised based on the injection of microbubbles..$^{4-9}$ Despite the less invasive procedure, it still requires the injection of microbubbles. Furthermore, it only provides a short time window for imaging as the bubbles are taken up by the liver or ruptures due to the acoustic pressure field produced by the ultrasound transducer.

In 1976, Holen et al. ${ }^{10}$ suggested a non-invasive method for estimating local pressure gradients by using the peak systolic blood velocity measured from ultrasound data. The velocity was used as input to a modified version of the orifice equation introduced by Golin ${ }^{11}$ that related the peak velocity to the pressure gradient along the region of interest. This non-invasive assessment of the pressure gradients, however, suffers from major flaws as it is solely dependent on a single velocity estimate and does not take complex flow patterns into account. Deducing a pressure gradient based on a single velocity estimate makes it sensitive to a series of unwanted hemodynamic factors that are uncorrelated to the actual constrictions effect on the peak velocity, e.g. abnormal cardiac output caused by cardiomyopathy. Hence, a more refined method for estimating the pressure gradients was suggested in 2003 by Ohtsuki and Tanake. ${ }^{12}$ This method also relied on ultrasound data, but the estimated pressure gradients were instead based on a simplified version of the Navier-Stokes equation. The Navier-Stokes equation describes 
the motion of fluids and provides easy access to information regarding the temporal and spatial pressure variations in the system, making it suitable for time-varying flow phenomena having complex features. Ohtsuki and Tanake assumed the viscosity to be negligible along with the external forces on the system. In addition, they assumed that the velocity normal to the scan plane was zero. The suggested method uses the axial velocity component found from spectral Doppler to deduce a measurement of the transverse velocity component and then inputs both velocity components in the Navier-Stokes equation. Applying the two in-plane velocity components to the Navier-Stokes equation offers the advantage of including spatial pressure variations into the estimator, unlike the method suggested by Holen et al. ${ }^{10}$ A shortcoming of the method suggested by Ohtsuki and Tanake ${ }^{12}$ is that the estimated pressure is solely based on the detected axial velocity, making the estimator inadequate for flow parallel to the ultrasound transducer. The method cannot measure the flow perpendicular to the beam propagation direction independently of angle and the axial velocity, which makes it insufficient for estimating pressure gradients in complex flow phenomena, such as vortexes that are common in hemodynamics.

Magnetic resonance imaging (MRI) is able to produce simultaneous velocity measurements in all three spatial directions $^{13}$, which enables derivation of pressure gradients without uncertainties related to the assumptions of out-of-plane motion. ${ }^{14}$ Most examples in the literature on deducing pressure gradients from MRI data are derived using the Navier-Stokes equation. ${ }^{14-17}$ The high spatial resolution in MRI forms a good foundation for calculating the convective acceleration used in the estimator. However, the temporal resolution is poor compared to ultrasound imaging. Often the temporal variation has to be acquired from several hundred cardiac cycles through ECG gating. This poses a significant downside to MRI, when performing pressure measurements on dynamic flow systems such as the cardiovascular system. Furthermore, MRI requires a long acquisition time when performing velocity measurements and is far more expensive than ultrasound imaging. A non-invasive alternative that addresses the mentioned issue is, thus, needed.

This paper presents pressure gradients estimated from ultrasound vector velocity data by exploiting the Transverse Oscillation (TO) method developed by Jensen and Munk ${ }^{18}$ and Anderson ${ }^{19}$ who suggested a similar approach. The TO method is unlike the method proposed by Ohtsuki and Tanake ${ }^{12}$ able to estimate the two spatial velocity components within the ultrasound scan plane independently of each other. This allows for determination of for examples flow vortices or other complex features that are likely to be present in a constricted flow pattern. The method employed for estimating the gradients have initially been suggested by Henze. ${ }^{20}$ The purpose of this paper is to compare pressure gradients derived from ultrasound data to pressure gradients obtained using fluid-simulation software. The idea is to show that pressure gradients can be measured non-invasively using ultrasound to replace or supplement catheterization. The presented results are all obtained from data recorded on a flow model that imitates a constricted carotid artery. The studies are conducted for a constant-flow system to verify the feasibility of the suggested method. Pressure gradients are estimated from ultrasound vector velocities using the Navier-Stokes equation and compared to a finite element simulation of the flow model. The geometry of the flow simulation model is reproduced using MRI data, thereby providing identical flow domains in measurement and simulation.

\section{ESTIMATING PRESSURE GRADIENTS}

The following section presents the method for calculating pressure gradients based on the Navier-Stokes equation using ultrasound vector velocity data.

The Navier-Stokes equation for an isotropic incompressible Newtonian fluid is given by

$$
\rho\left[\frac{\partial \mathbf{v}}{\partial t}+\mathbf{v} \cdot \nabla \mathbf{v}\right]=-\nabla p+\rho \mathbf{g}+\mu \nabla^{2} \mathbf{v}
$$

The equation describes the development of fluid velocity $\mathbf{v}\left(v_{x}, v_{y}, v_{z}\right)$ by relating the body forces acting on the isotropic fluid volume to its acceleration and density, where $\rho$ is the density of the fluid and $\mu$ its viscosity. The left-hand terms sum the local $\frac{\partial \mathbf{v}}{\partial t}$ and convective acceleration $\mathbf{v} \cdot \nabla \mathbf{v}$ of the velocity, where $\nabla$ is the spatial differential operator $\left(\frac{\partial}{\partial x}, \frac{\partial}{\partial y}, \frac{\partial}{\partial z}\right)$. The right-hand side sum-up the surface and volume forces, which include the desired pressure gradient $-\nabla p$, as well as a gravitational force $\mathbf{g}$ acting on the fluid volume and a viscous drag caused by the viscosity of the fluid $\mu \nabla^{2} \mathbf{v}$, where $\nabla^{2} \mathbf{v}$ is the Laplacian of the velocity. For clinical applications, the 
effect of the viscous term in (1) can be omitted as this has no significant influence on flow in larger vessels. ${ }^{21,22}$ A patient undergoing an ultrasound scan is usually placed in a horizontal position, so the gravitational term can be neglected as well. The pressure gradient is, thus, directly linked to the acceleration field of the fluid

$$
\nabla p=-\rho\left[\frac{\partial \mathbf{v}}{\partial t}+\mathbf{v} \cdot \nabla \mathbf{v}\right]
$$

As the conducted study considers flow that is in-variant with respect to time, no changes in the transient acceleration occur, thus

$$
\nabla p=-\rho[\mathbf{v} \cdot \nabla \mathbf{v}] .
$$

\subsection{Implementation the estimator on ultrasound data}

Equation (3) states that all three vector components of $\mathbf{v}\left(v_{x}, v_{y}, v_{z}\right)$ must be known to estimate the pressure gradient $-\nabla p$. Using the TO approach the scanner is able to measure the two-dimensional in-plane velocity vector $^{23,24} \mathbf{v}=\left(v_{x}, 0, v_{z}\right)$. The method is therefore developed assuming that the out-of-plane velocity $v_{y}$ is zero. Though, the method can easily be extended to use full three-dimensional data.

Assuming that $v_{y}$ is zero makes two-dimensional pressure gradients obtainable in the presented set-up. The in-plane vector components of the pressure gradients are expressed here in rectangular coordinates:

$$
\left[\begin{array}{c}
\frac{\partial p}{\partial x} \\
\frac{\partial p}{\partial z}
\end{array}\right]=-\rho\left[\begin{array}{c}
v_{x} \frac{\partial v_{x}}{\partial x}+v_{z} \frac{\partial v_{x}}{\partial z} \\
v_{x} \frac{\partial v_{z}}{\partial x}+v_{z} \frac{\partial v_{z}}{\partial z}
\end{array}\right]
$$

The derivatives in (4) are calculated from vector velocity ultrasound data using a finite central difference approximation. ${ }^{25}$ The in-plane pressure gradient components are here calculated discretely for each position in the scan plane:

$$
\frac{\Delta \mathbf{P}(i, j)}{\Delta x} \simeq-\rho\left[\begin{array}{l}
\mathbf{V}_{x}(i, j) / 2 \Delta x \\
\mathbf{V}_{z}(i, j) / 2 \Delta z
\end{array}\right] \cdot\left[\begin{array}{c}
\mathbf{V}_{x}(i-1, j)-\mathbf{V}_{x}(i+1, j) \\
\mathbf{V}_{x}(i, j-1)-\mathbf{V}_{x}(i, j+1)
\end{array}\right]
$$

and

$$
\frac{\Delta \mathbf{P}(i, j)}{\Delta z} \simeq-\rho\left[\begin{array}{l}
\mathbf{V}_{x}(i, j) / 2 \Delta x \\
\mathbf{V}_{z}(i, j) / 2 \Delta z
\end{array}\right] \cdot\left[\begin{array}{l}
\mathbf{V}_{z}(i-1, j)-\mathbf{V}_{z}(i+1, j) \\
\mathbf{V}_{z}(i, j-1)-\mathbf{V}_{z}(i, j+1)
\end{array}\right]
$$

respectively. Here $\mathbf{V}_{x}, \mathbf{V}_{z}$ and $\frac{\Delta \mathbf{P}}{\Delta x}, \frac{\Delta \mathbf{P}}{\Delta z}$ denote two-dimensional fields of discrete vector velocity components and pressure gradient components, respectively. The position in the field is given by $(i, j)$, while $\Delta x$ and $\Delta z$ is the sampling interval of the velocity field in the axial and lateral direction, respectively. The calculated pressure gradients are low-pass filtered using a normalized Hann window $\mathcal{H}_{2 D}$ to reduce noise from the numerical differentiation. The 2-D window is designed as:

$$
\mathcal{H}_{2 D}=0.5\left(1-\cos \left(2 \pi \frac{l^{\prime}}{L-1}\right)\right) 0.5\left(1-\cos \left(2 \pi \frac{l}{L-1}\right)\right),
$$

where 1 is a vector of integers from 0 to L- 1 and $\mathrm{L}$ is the length of the window. The calculated gradient fields are filtered by convolving their individual vector components with the normalized Hann window as:

$$
\frac{\Delta \mathbf{P}_{F}(i, j)}{\Delta x}=\frac{\mathcal{H}_{2 D}}{\sum_{r, s=1}^{N} \mathcal{H}_{2 D}(r, s)} * \frac{\Delta \mathbf{P}(i, j)}{\Delta x} \quad \text { and } \quad \frac{\Delta \mathbf{P}_{F}(i, j)}{\Delta z}=\frac{\mathcal{H}_{2 D}}{\sum_{r, s=1}^{N} \mathcal{H}_{2 D}(r, s)} * \frac{\Delta \mathbf{P}(i, j)}{\Delta z} .
$$


Table 1: Measurement settings for acquiring data

\begin{tabular}{lll}
\hline \hline & Parameter & Value \\
\hline BK Scanner & Transducer & Linear array 8670 \\
& Mode & Carotid TVI \\
& Pulse repetition frequency & $1.3 \mathrm{kHz}$ \\
& Frame rate & $18 \mathrm{~Hz}$ \\
& Centre frequency & $5 \mathrm{MHz}$ \\
& No. frames & 50 \\
Flow setup & Volumetric flow rate & $1.0 \mathrm{~cm} / \mathrm{s}($ constant) \\
& Inner radius & $0.6 \mathrm{~cm}$ \\
& Constriction & $70 \%$ \\
& Depth to constriction & $2.3 \mathrm{~cm}$ \\
& Fluid density & $1,037 \mathrm{~kg} / \mathrm{m}^{3}$ \\
& Viscosity & $4.1 \cdot 10^{-3} \mathrm{~Pa} \cdot \mathrm{s}$ \\
\hline \hline
\end{tabular}

\section{DATA ACQUISITION}

The following section describes the data acquisition for estimating and validating the pressure gradients during constant flow conditions.

A BK Medical 2202 UltraView Pro Focus scanner (BK Medical, Herlev, Denmark) equipped with an UA2227 research interface ${ }^{26}$ is used for obtaining ultrasound RF data. A C70-SSEA flow phantom (Shelley Medical Imaging Technologies, Toronto, Canada) is scanned using a BK8670 linear array transducer at 18 frames per second. The flow phantom is a model of a carotid artery having a constriction at the beginning of the internal carotid artery that reduces the cross-sectional area of the lumen by $70 \%$. The flow phantom is embedded in agar to mimic the characteristics of human tissue, and it is encased in an air tight acrylic box with an acoustic window making it compatible for ultrasound imaging as well as MRI. A constant flow profile driven by a CompuFlow 1000 Flow system (Shelley Medical Imaging Technologies, Toronto, Canada) is set for the phantom at a flow rate of $1.0 \mathrm{ml} / \mathrm{s}$. Blood mimicking fluid is used with a density of $1,037 \mathrm{~kg} / \mathrm{m}^{3}$ and a viscosity of $4.1 \mathrm{mPa} \cdot \mathrm{s}$. A summary of the acquisition settings is listed in Table 1.

The reliability of the produced pressure gradients is evaluated through comparison to a finite element model. The geometry of this model is constructed from MRI data of the flow phantom performed at the Department of Diagnostic Radiology at Rigshospitalet, Denmark, using a 3 Tesla Magnetom Trio scanner (Siemens, Munich, Germany). The MRI data are loaded into the commercial segmentation-software, ScanIP (Simpleware Ltd. Exeter, UK), which is used to construct the skeleton of the phantom's fluid domain. The constructed skeleton is exported to Comsol (v4.2a, Stockholm, Sweden) as a mesh of tetrahedrons. The mesh is smoothed by a Gaussian filter (dimensions: $1.00 \times 0.01 \times 0.01 \mathrm{~mm}$ ) to avoid any inexpedient sharp edges that otherwise could lead to computational divergence. Such sharp edges occur, if the spatial resolution in the scan images is smaller than the resolution required by Comsol. The mesh consists of more than 600,000 tetrahedrons that are especially dense in the constricted area of the phantom to ensure a high spatial resolution in the this particular region (mesh size: $0.15 \mathrm{~mm}$ ). A summary of the settings used for creating the geometry of the simulation model is listed in Table 2 .

\section{DERIVATION OF PRESSURE GRADIENTS}

The following section describes how pressure gradient maps are estimated based on the vector velocity estimates.

A vector velocity map of the flow through the constricted region of the phantom is estimated using the ultrasound vector velocity data. They are calculated by employing the TO method as described by Jensen and Munk $^{18}$ and by using the TO estimation method, ${ }^{27}$ yielding the two in-plane velocity components of the ultrasound beam. A low-pass filter is applied to the velocity estimates before calculating the convective acceleration to strengthen the robustness of the estimates. The filter is designed as in (6) for $\mathrm{L}=5$, corresponding to an axial length of $0.07 \mathrm{~mm}$ and a lateral width of $0.58 \mathrm{~mm}$. Furthermore, the velocity estimates are interpolated to 
Table 2: Measurement settings for acquiring validation data

\begin{tabular}{lll}
\hline \hline & Parameter & Value \\
\hline Magnetic resonance & Scanner & Siemens Magnetom Trio \\
& Strength & 3.0 Tesla \\
& Scan sequence & TOF $3-\mathrm{D}$ Multi-slab \\
& Voxel size & $0.7 \times 0.7 \times 1.0 \mathrm{~mm}$ \\
ScanIP & Up-sampling & $(\mathrm{x}, \mathrm{y}, \mathrm{z})=(0.9,0.15,0.15) \mathrm{mm}$ \\
& Filter size & $(\mathrm{x}, \mathrm{y}, \mathrm{z})=(1.0,0.01,0.01) \mathrm{mm}$ \\
& Mesh size & $0.15 \mathrm{~mm}$ \\
\hline \hline
\end{tabular}

improve the visual quality of the image, yielding an increase in the number of data elements by a factor of three. As the study is conducted on a constant-flow system, every estimated velocity frame is roughly the same, thus the mean over 50 ultrasound frames is calculated and used for deriving the pressure gradients. The pressure gradients are then calculated using (5) and (7).

The imported Comsol-mesh forming the geometrical structure of the simulation model is assigned conditions that mimic the actual flow conditions in the experimental set-up. These conditions are listed in Table 3. Stationary laminar flow of an incompressible fluid is selected. The initial values of the fluids velocity field and its pressure is set to zero, while having a forced laminar flow condition at the inlet of the model. The peak velocity across the constriction is set to $0.15 \mathrm{~m} / \mathrm{s}$, equivalent to the velocity estimated from the ultrasound data.

The result of the suggested pressure gradient estimator is investigated by considering its average standard deviation $\hat{\sigma}$ and its bias $\hat{\mathbf{B}}$ in reference to the simulated data. They are calculated as:

$$
\hat{\sigma}=\frac{1}{\hat{P G_{\max }}} \sqrt{\frac{1}{N} \sum_{m=1}^{N} \boldsymbol{\sigma}_{t}^{2}(m)} \quad \text { and } \quad \hat{\mathbf{B}}=\frac{1}{\hat{P G_{\max } N}} \sum_{m=1}^{N} \boldsymbol{\mu}_{\text {est }}(m)-\boldsymbol{\mu}_{\text {sim }}(m)
$$

where $N$ is the number of data points along the spatial path that is investigated and $\boldsymbol{\mu}_{\text {est }}$ is the mean of these points. The standard deviation of the estimated gradient fields $\boldsymbol{\sigma}_{t}$ is averaged along the investigated path. The simulated data along the inspected path is denoted $\boldsymbol{\mu}_{\text {sim }}$. Both, standard deviation and bias are normalized to the peak mean estimated pressure gradient, $\hat{P G_{\max }}=\max \left(\boldsymbol{\mu}_{\text {est }}\right)$.

Table 3: Simulation settings in Comsol

\begin{tabular}{lll}
\hline \hline & Parameter & Value \\
\hline Study & Flow & Laminar \\
& Geometry & Three dimensional \\
& Stationary solver & Direct, MUMPS \\
Fluid properties & Compressibility & Incompressible \\
& Density & $1,037 \mathrm{~kg} / \mathrm{m}^{3}$ \\
& Viscosity & $4.1 \cdot 10^{-3} \mathrm{~Pa} \cdot \mathrm{s}$ \\
Initial conditions & Velocity field & $(\mathrm{x}, \mathrm{y}, \mathrm{z})=(0,0,0) \mathrm{m} / \mathrm{s}$ \\
& Pressure & $0 \mathrm{~Pa}$ \\
Inlet conditions & Inflow & $\mathrm{Laminar}$ \\
& Flow rate & $2 \mathrm{~cm}{ }^{3} / \mathrm{s}$ \\
& Entrance length & $1 \mathrm{~m}$ \\
Outlet conditions & Outflow & Pressure, no viscous stress \\
& Pressure & $0 \mathrm{~Pa}$ \\
Wall & Condition & $\mathrm{No}-\mathrm{slip}$ \\
\hline \hline
\end{tabular}




\section{RESULTS}

The following section presents the results of the pressure gradient estimator. The results are based on vector velocity data acquired using a commercial ultrasound scanner at 18 frames per second. The presented results are all captured from the carotid flow phantom at the site of the constriction, shown in Fig. 1. It also shows the mesh of the simulation model superimposed onto the B-mode image. The estimated and simulated results will throughout the section be discussed as they are presented.

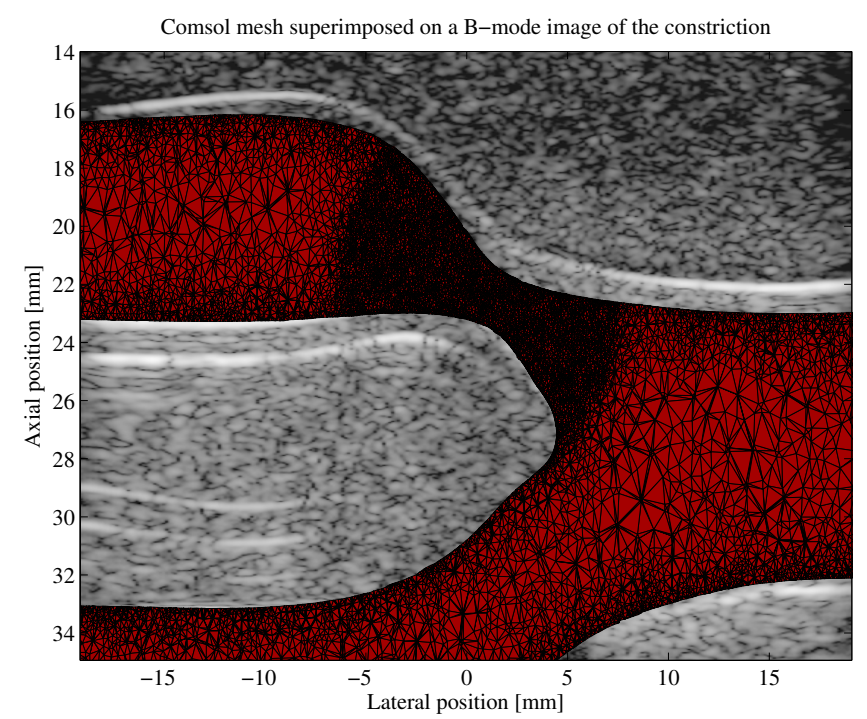

Figure 1: B-mode image of the carotid flow phantom at the site of the constriction plotted along with the mesh skeleton of the simulation model.

\section{Presentation of the pressure gradients}

A vector velocity map of the flow through the constricted area of the phantom is plotted in Fig. 2 along with the estimated and simulated pressure gradients. The fluid flows from right to left and reaches its peak velocity at the center of the constriction with $0.15 \mathrm{~m} / \mathrm{s}$. The center plot in Fig. 2 shows the estimated pressure gradients that are calculated using the velocities in the left plot. The plot to the right shows the simulated pressure gradients.

The background color indicates the magnitude of the arrows, while these show the direction and size of either the fluid velocity or of the pressure gradients. The two gradient plots display arrows that tend to point away from the center of the constriction, indicating that a low pressure is present here, as a gradient always point in direction of increasing values. The white-dotted lines on the center plot and on the right plot indicates the positions used for the quantitative plots shown in the next section.

\section{Quantitative results of pressure gradient estimator}

Quantitative results of the pressure gradient estimator are obtained by investigating its bias and standard deviation. These are calculated along the dotted lines in the two plots to the right of Fig. 2 for the axial and lateral component of the gradients.

Fig. 3 shows the mean magnitude of the axial and lateral pressure gradients along the longitudinal direction for 50 frames. It also shows the range of one standard deviation given at a frame rate of $9 \mathrm{~Hz}$. The blue line shows the simulated pressure gradients along the same path.

The estimator produced pressure gradients of magnitudes varying from $0 \mathrm{kPa} / \mathrm{m}$ to $10 \mathrm{kPa} / \mathrm{m}$. It has a normalized bias of $-9.1 \%$ for the axial component and $-7.9 \%$ for the lateral component with a standard deviation given in reference to the peak gradient of $28.4 \%$ and $64.5 \%$, respectively. Increasing the number of velocity frames used in the estimator will improve the standard deviation of the estimates. Fig. 4 shows the effect of this by plotting the standard deviation as a function of averaging-time used by the estimator to produce a single two-dimensional 

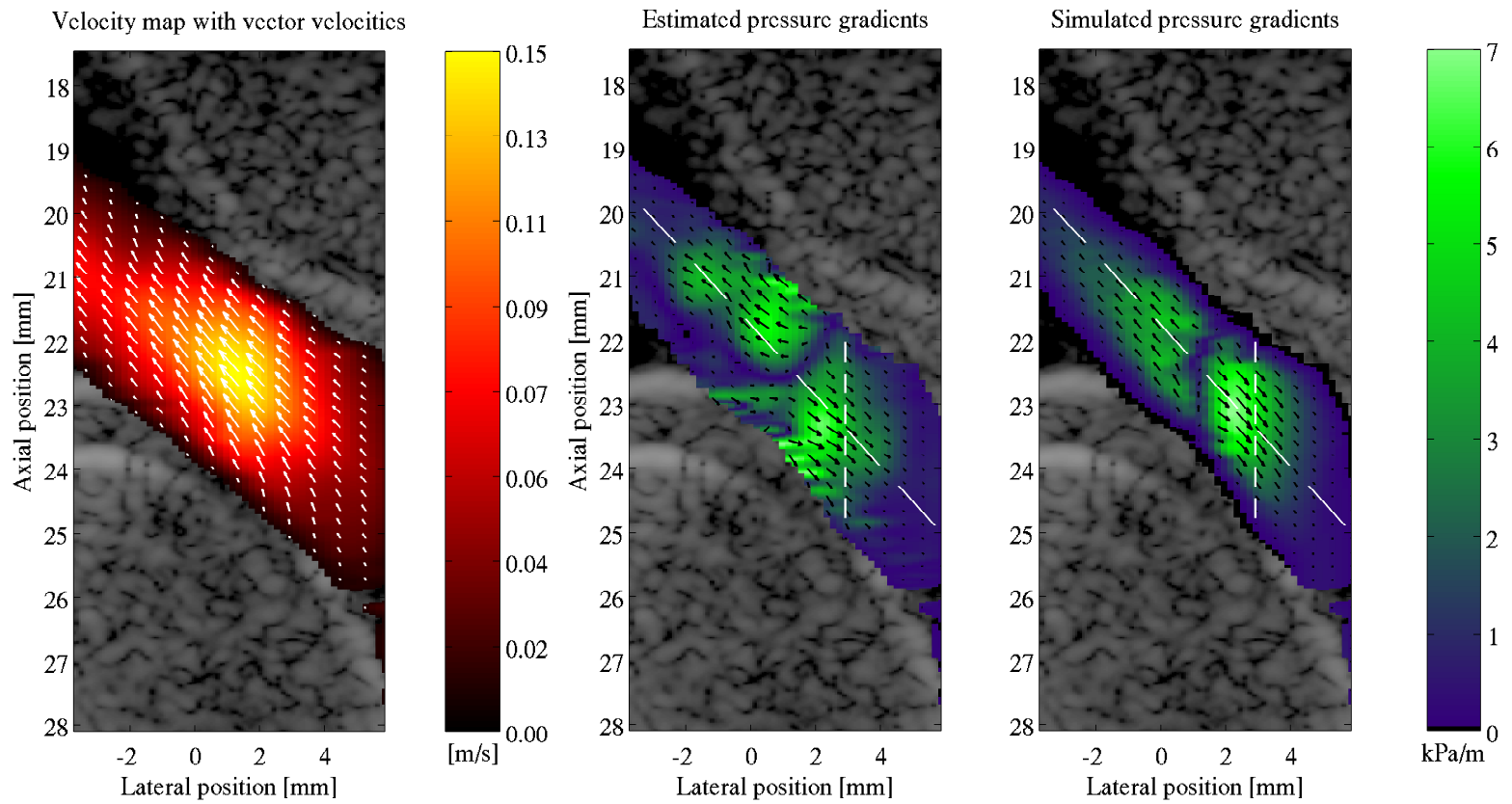

Figure 2: Vector velocity map along with the estimated and simulated pressure gradients. The plot to the left shows a vector velocity map of the blood mimicking fluid that flows through the constriction from right to left. The center plot show the pressure gradients estimated from vector velocity data, while the figure to the right show the simulated gradients.
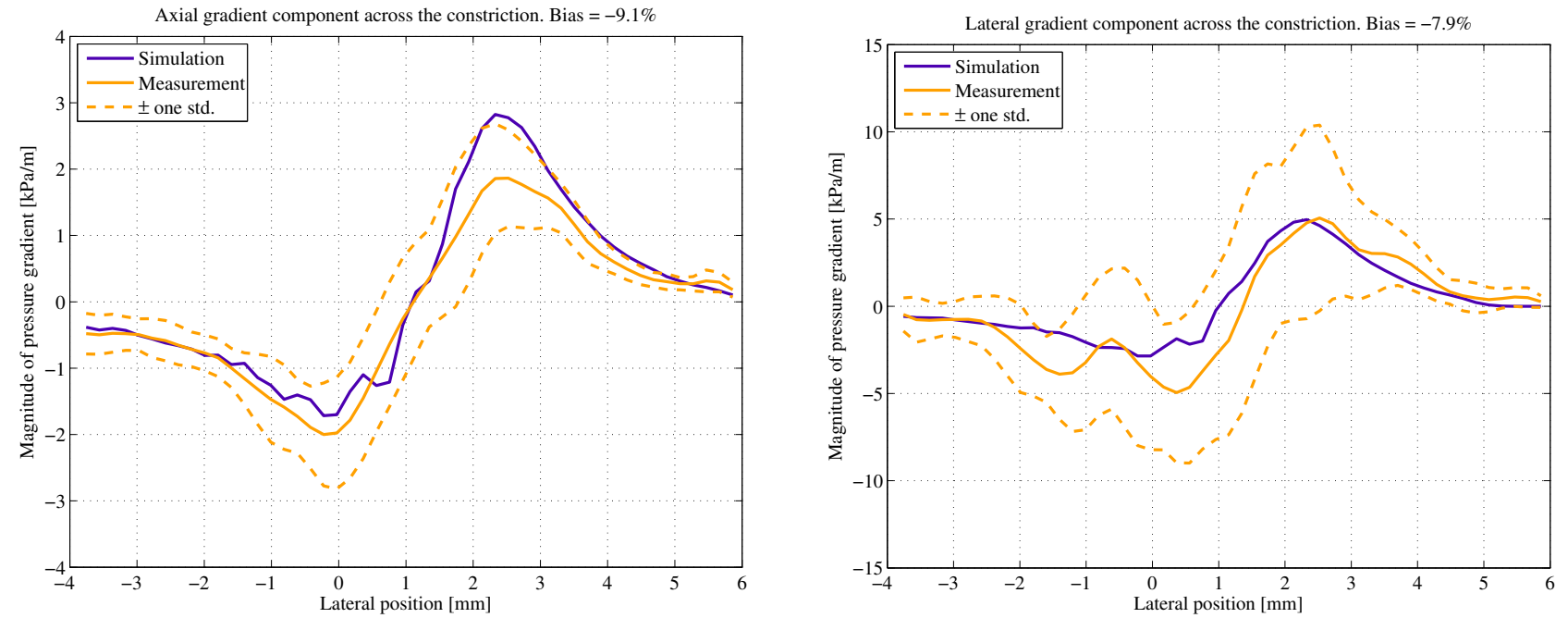

Figure 3: Estimated and simulated pressure gradients along the longitudinal direction of the constriction. The two graphs show the axial and lateral component of the pressure gradient, respectively.

pressure gradient field. More velocity frames are averaged as the time increases, making the estimates more robust in a noisy environment. However, the improved standard deviation is at the expense of temporal resolution as the estimators true frame rate drops proportional to the increase in averaging-time.

Quantitative results along the vertical white-line in Fig. 2 are presented in Fig. 5. The graphs in Fig. 5 present magnitudes of pressure gradient varying from $0 \mathrm{kPa} / \mathrm{m}$ to $7 \mathrm{kPa} / \mathrm{m}$. A normalized bias was found for the axial component of $-5.7 \%$ and for the lateral component it was $13.9 \%$ with a standard deviation of $45.2 \%$ and $83.2 \%$. The spikes in the standard deviation seen to the right of the lateral position $24 \mathrm{~mm}$, are artefacts caused by boundary effects. These artefacts can also be spotted at the vessel wall in the center plot of Fig.2. 


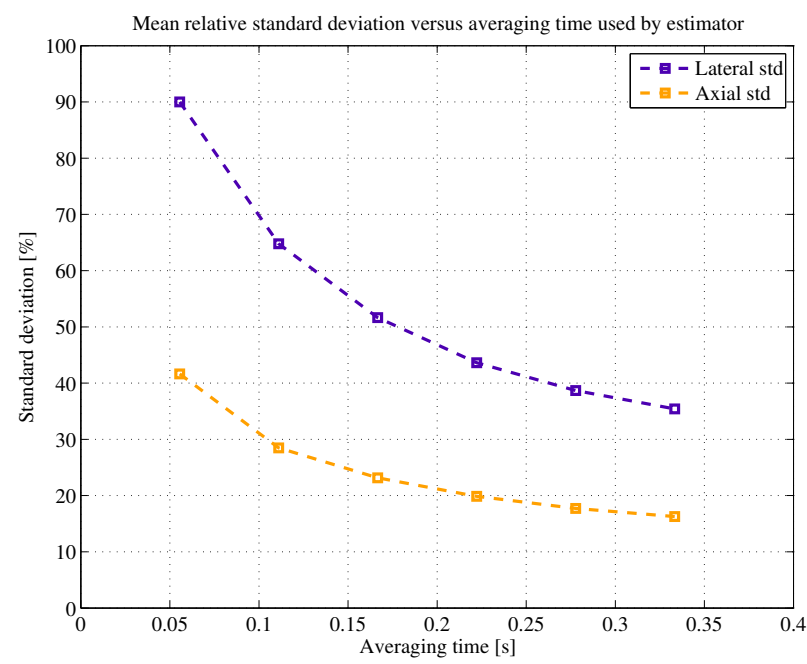

Figure 4: Mean standard deviation of the estimator given as a function of averaging-time used by the estimator to produce a single two-dimensional pressure gradients field.
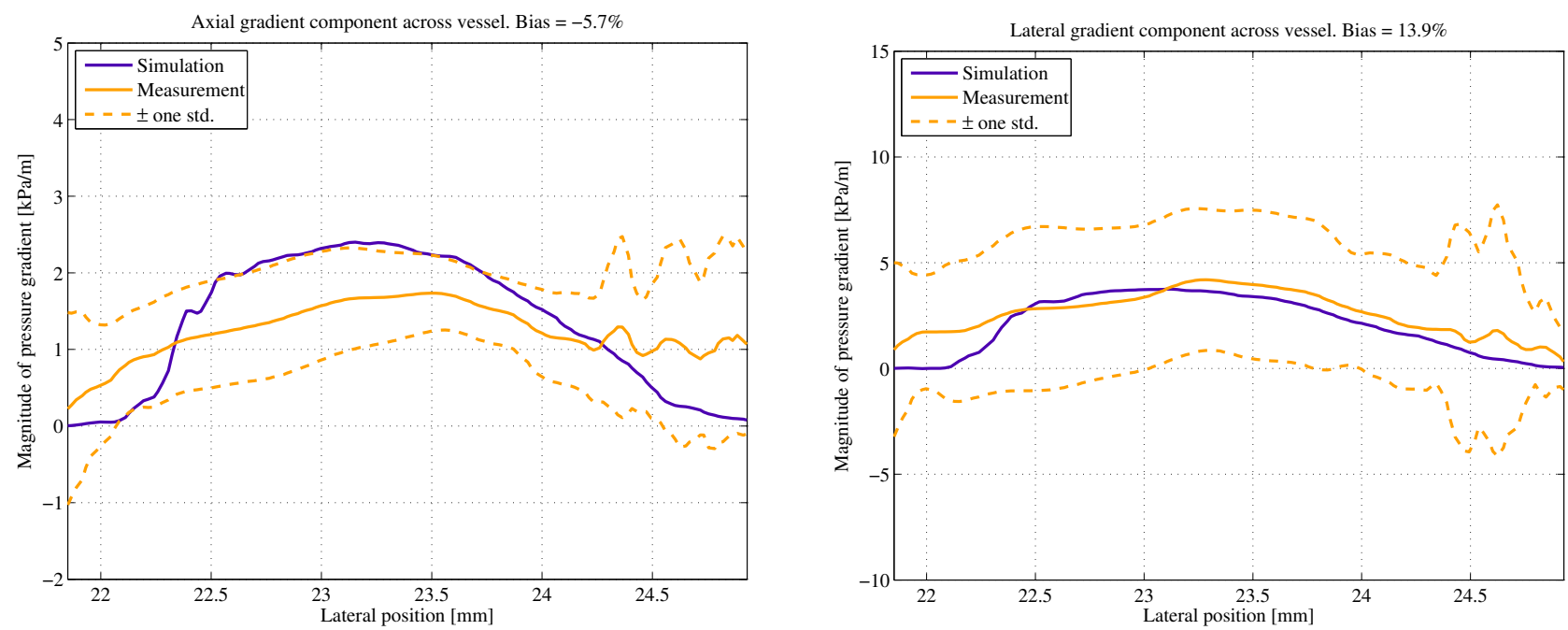

Figure 5: Estimated and simulated pressure gradients along the cross-sectional direction of the constriction. The two graphs show the axial and lateral component of the pressure gradient, respectively.

\section{DISCUSSION}

A non-invasive method for estimating pressure gradients using ultrasound vector velocity data has been presented. The method has managed to produce pressure gradients within fluid flow that has a maximum bias of $-9.1 \%$ and $13.9 \%$ in the axial and lateral direction, respectively. The standard deviation of the estimator varied from $24.4-42.2 \%$ in the axial direction and from $64.5-83.2 \%$ in the lateral direction.

Fig. 2 shows pressure gradients that tend to point away from the area of high velocities. This observation is in accordance with expectations as there exist a clear physical relationship between fluids pressure and its velocities. A way of clarifying this relationship is to consider the energy of the flow system for which no energy is exchanged between the fluid and the vessel walls, meaning that the energy level is constant throughout the system. The total energy is composed by the sum of potential energy and kinetic energy. Since the gravitational force can be neglected, the only force giving rise to the potential energy is pressure. As the fluid flows into the constriction it starts to accelerate to keep up a constant flow rate and the kinetic energy increases. Since no energy is put into the flow system from its surroundings, the increase in kinetic energy must come from the potential energy, thereby decreasing the pressure in areas of high velocities. As the fluid flows past the constriction it decelerates, 
turning kinetic energy back into potential energy. This explains why the gradients tend to point away from the site of the highest velocities as pressure most be lowest there. The assumption of a constant energy level is of course not entirely true, since energy dissipation will occur as the fluid is not inviscid. However, the amount of energy dissipation is very limited in reference to the total change in energy that exist along the constriction.

No previous studies on the subject have managed to estimate and validate 2-D pressure gradients from vector velocity data obtained using the TO approach. Unlike the method suggested by Holen et al., ${ }^{10}$ this method is able to take convective fluid accelerations into consideration making it suitable for more complex flow phenomena. Every term in the estimator is calculated relative to neighboring velocity estimates, yielding gradients that are independent of systemic flow factors, such as abnormalities in the cardiac output. The proposed method also offers the advantage of including temporal pressure changes, however, this was not considered in this paper as constant flow was studied. Yet, being able to include temporal changes is essential for studies on the cardiovascular system. Such studies usually require a frame rate of approximately $20 \mathrm{~Hz}$. Furthermore, the method can easily be extended to use full three-dimensional data. The estimator presented in this study produced images of pressure gradients at a frame rate of $9 \mathrm{~Hz}$, since two velocity frames, sampled at $18 \mathrm{~Hz}$, were used for calculating one frame of pressure gradients. A way of increasing this frame rate, while maintaining the presented precision, is to shrink the width of the colorbox used during the acquisition of velocity ultrasound data, as fewer pulses are required for covering a narrower region. A reason for the high standard deviation is found in the poor spatial resolution of ultrasound imaging, which gives rise to a significant noise contribution during the numerical differentiation of the velocity fields. However, the lack in spatial resolution can be considered counterbalanced by the temporal resolution, which is one of the most significant advantages in ultrasound imaging compared to MRI. The relative high temporal resolution is the result of a low data acquisition time enabling the possibilities of producing real-time pressure gradient images using ultrasound. This will give the physician a tool for viewing intra-vascular changes in pressure as they occur, for example during a cardiac cycle or changes observed over several cycles. More importantly, the physician would no longer have to insert catheters into the blood vessel to obtain a map of the pressure changes. A shortcoming of the proposed method is its lacking ability to estimate the out-of-plane velocity component $v_{y}$. This makes the estimator vulnerable in complex flow environments that has vector velocity components in all three spatial direction. However, Pihl and Jensen ${ }^{28,29}$ recently presented a novel technique for measuring the out-of-plane velocity using an ultrasound system. Exploiting this technique, would yield three-dimensional vector velocity fields that could easily be included in the suggested pressure gradient estimator, leaving no-need for assuming that $v_{y}$ is zero.

\section{CONCLUSION}

A non-invasive method for deriving pressure gradients using vector velocity ultrasound data has been presented. The pressure gradients were derived using the Navier-Stokes equation for incompressible fluids. The vector velocities inserted into the equation were estimated using the TO method yielding the two in-plane velocity components. The obtained pressure gradients were evaluated by comparison to a finite element simulation model, which geometry was obtained from MRI data. The employed method has successfully managed to produce pressure gradients within a constricted flow phantom of magnitudes varying from $0 \mathrm{kPa} / \mathrm{m}$ to $10 \mathrm{kPa} / \mathrm{m}$. Proof of concept studies conducted along the longitudinal direction of a constricted flow phantom showed a normalized bias of $-9.1 \%$ for the axial component and $-7.9 \%$ for the lateral component with precision given in reference to the peak gradient of $28.4 \%$ and $64.5 \%$, respectively. Studies made across the constriction were also conducted giving a normalized bias of $-5.7 \%$ for the axial component and $13.9 \%$ for the lateral component at a precision of $45.2 \%$ and $83.2 \%$.

\section{ACKNOWLEDGMENTS}

The authors would like the thank Jesper Rohde at BK Medical, Denmark, for putting their flow phantom at our disposal and Carsten Thomsen at Rigshospitalet, Denmark, for performing the MRI scans. The presented work has been financially supported by grant 024-2008-3 from the Danish Advanced Technology Foundation, BK Medical Aps, Denmark and Radiometer A/S, Denmark. 


\section{REFERENCES}

[1] Baim, D. S. and Grossman, W., [Grossman's cardiac catheterization, angiography, and intervention], Lippincott Williams \& Wilkins publishers (2000).

[2] Skinner, J. S. and Adams, P. C., "Outpatient cardiac catheterisation," Int. J. Cardiol. 53, 209-219 (March 1996).

[3] Fairbank, W. M. and Scully, M. O., "A new noninvasive technique for cardiac pressure measurements: resonant scattering of ultrasound from bubbles," IEEE Trans. Biomed. Eng. 24, 107-110 (1977).

[4] Bouakaz, A., Frinking, P. J., de Jong, N., and Bom, N., "Noninvasive measurement of the hydrostatic pressure in a fluid-filled cavity based on the disappearance time of micrometer-sized free gas bubbles," Ultrasound Med. Biol. 25, 1407-1415 (1999).

[5] Shi, W. T., Forsberg, F., Raichlen, J. S., and Needleman, L., "Pressure dependence of subharmonic signals from contrast microbubbles," Ultrasound Med. Biol. 25, 275-283 (1999).

[6] Adam, D., Sapunar, M., and Burla, E., "On the relationship between encapsulated ultrasound contrast agent and pressure," Ultrasound Med. Biol. 31, 673-686 (2005).

[7] Forsberg, F., Liu, J.-B., Shi, W. T., Furuse, J., Shimizu, M., and Goldberg, B. B., "In vivo pressure estimation using subharmonic contrast microbubble signals: Proof of concept," IEEE Trans. Ultrason., Ferroelec., Freq. Contr. 52(4), 581-583 (2005).

[8] Andersen, K. S. and Jensen, J. A., "In vitro measurement of ambient pressure changes using a realistic clinical setup," in [Proc. IEEE Ultrason. Symp.], 1096-1099 (2008).

[9] Forsberg, F., Dave, J., Halldorsdottir, V. G., Leodore, L. M., Lin, F., Hall, A. L., and Thomenius, K., "Applying real-time noninvasive pressure estimation obtained from subharmonic contrast microbubble signals," Proc. IEEE Ultrason. Symp. , 1694-1697 (2008).

[10] Holen, J., Aaslid, R., and Landmark, K., "Determination of pressure gradient in mitral stenosis with a non-invasive ultrasound Doppler technique," Acta med. scand. 32, 455-460 (1976).

[11] Gorlin, R. and Gorlin, S. G., "Hydraulic formula for calculation of the area of the stenotic mitral valve, other cardiac valves, and central circulatory shunts," Am. Heart J. 41, 1-29 (January 1951).

[12] Ohtsuki, S. and Tanaka, M., "Doppler pressure field deduced from the Doppler velocity field in an observation plane in a fluid," Ultrasound Med. Biol. 29(10), 1431-1438 (2003).

[13] Kilner, P. J., Yang, G. Z., Mohiaddin, R. H., Firmin, D. N., and Longmore, D. B., "Helical and retrograde secondary flow patterns in the aortic arch studied by three-directional magnetic resonance velocity mapping," Circulation 88(5), 2235-2247 (1993).

[14] Bock, J., Frydrychowicz, A., Lorenz, R., Hirtler, D., Barker, A. J., Johnson, K. M., Arnold, R., Burkhardt, H., Hennig, J., and Markl, M., "In vivo noninvasive 4D pressure difference mapping in the human aorta: phantom comparison and application in healthy volunteers and patients," Magn. Reson. Med. 66(4), 1079-1088 (2011).

[15] Tasu, J. P., Mousseaux, E., Delouche, A., Oddou, C., Jolivet, O., and Bittoun, J., "Estimation of pressure gradients in pulsatile flow from magnetic resonance acceleration measurements," Magn. Reson. Med. 44(1), 66-72 (2000).

[16] Thompson, R. B. and McVeigh, E. R., "Fast measurement of intracardiac pressure differences with 2D breath-hold phase-contrast MRI," Magnetic Resonance in Medicine 49(6), 1056-1066 (2003).

[17] Herment, A., Besson, G., Pellot-Barakat, C., and Frouin, F., "Estimation of pressure gradient images from velocity encoded MR acquisitions," Computers in Cardiology 35, 933-936 (2008).

[18] Jensen, J. A. and Munk, P., "A New Method for Estimation of Velocity Vectors," IEEE Trans. Ultrason., Ferroelec., Freq. Contr. 45, 837-851 (1998).

[19] Anderson, M. E., "Multi-dimensional velocity estimation with ultrasound using spatial quadrature," IEEE Trans. Ultrason., Ferroelec., Freq. Contr. 45, 852-861 (1998).

[20] Henze, L., Ultrasound flow estimation, Master's thesis, Dept. of Elec. Eng., Technical University of Denmark, Lyngby, Denmark (2008).

[21] Prandtl, L., [Essentials of Fluid Dynamics], London: Blackie \& Son (1952).

[22] Wood, N. B., "Aspects of fluid dynamics applied to the large arteries," J. Theor. Biol. 199, 137-161 (April 1999). 
[23] Evans, D. H., Jensen, J. A., and Nielsen, M. B., "Ultrasonic colour Doppler imaging," Interface Focus 1, 490-502 (August 2011).

[24] Hansen, P. M., Pedersen, M. M., Hansen, K. L., Nielsen, M. B., and Jensen, J. A., "Demonstration of a vector velocity technique," Ultraschall in Med 32, 213-5 (2011).

[25] Smith, G. D., [Numerical Solution of Partial Differential Equations: Finite Difference Methods], Oxford University Press (1985).

[26] Hemmsen, M. C., Nikolov, S. I., Pedersen, M. M., Pihl, M. J., Enevoldsen, M. S., Hansen, J. M., and Jensen, J. A., "Implementation of a versatile research data acquisition system using a commercially available medical ultrasound scanner," IEEE Trans. Ultrason., Ferroelec., Freq. Contr. 59(7), 1487-1499 (2011).

[27] Jensen, J. A., "A New Estimator for Vector Velocity Estimation," IEEE Trans. Ultrason., Ferroelec., Freq. Contr. 48(4), 886-894 (2001).

[28] Pihl, M. J. and Jensen, J. A., "3D velocity estimation using a 2D phased array," in [Proc. IEEE Ultrason. Symp.], 430-433 (2011).

[29] Pihl, M. J. and Jensen, J. A., "Measuring 3D velocity vectors using the transverse oscillation method," in [Proc. IEEE Ultrason. Symp.], IEEE (2012). 